Original article

Paediatrics Today 2016;12(1):139-148

DOI $10.5457 / \mathrm{p} 2005-114.150$

\title{
ABDOMINAL ULTRASOUND IN CHILDREN - ARE WE CAREFUL ENOUGH WHEN IT COMES TO LUNG BASES?
}

\author{
Jovan LOVRENSKI ${ }^{*}$, Dajana POPADIĆ ${ }^{2}$, Ivan VARGA ${ }^{1}$, Slobodanka PETROVIĆ ${ }^{3}$
}

${ }^{1}$ Radiology Department, Faculty of Medicine, University of Novi Sad, Novi Sad Serbia, ${ }^{2}$ Faculty of Medicine, University of Novi Sad, Novi Sad, Serbia, ${ }^{3}$ Pulmonology Department, Faculty of Medicine University of Novi Sad, Novi Sad, Serbia

Correspondence: jovan.lovrenski@mf.uns.ac.rs Tel.: + 381641436030

Fax.: + 38121425509

Received: Mart 26, 2016

Accepted: June 13, 2016

Key words: Ultrasound - Abdomen • Children - Pneumonia - Abdominal pain.

\begin{abstract}
Objective - To investigate the frequency of positive findings within the lung bases during abdominal ultrasound (US) examinations in children with referral diagnosis not suggesting pulmonary disease. Materials and methods - A retrospective study included all children with abdominal US performed within 2 years by a single pediatric radiologist in the regional children's hospital. Of all examined children, 22 (mean $7.4 \mathrm{y}, \mathrm{SD}=4.2 \mathrm{y}$ ) had pathological findings detected within the lung bases, without referral diagnosis suggesting a pulmonary pathology. Each abdominal US included a trans-abdominal (trans-hepatic and trans-splenic) approach to both lung bases. Results - While performing abdominal US a detected pulmonary pathology included: 19 pneumonias and 3 pleuropneumonias, with the most common referral diagnosis being abdominal pain ( 15 children), followed by nausea and vomitting ( 3 children), febrile convulsiones ( 2 children), exacerbation of juvenile arthritis (1), and a routine follow-up a day after hydrostatic reduction of ileocolic intussusception (1). In outpatients with referral diagnosis of abdominal pain, $0.95 \%$ had positive lung base US findings. Conclusion - Each radiologist should be aware of the potential pathological finding within the lung bases when performing abdominal ultrasound in children. Its detection might have a serious impact on the patient's treatment.
\end{abstract}

\section{Introduction}

Abdominal ultrasound (US) in children represents one of the most frequently used diagnostic radiological procedures in daily clinical practice, with the most common referral diagnosis being acute abdominal pain. The causes of acute abdominal pain in children which stand out are: constipation, mesenteric lymphadenitis, acute appendicitis, gastroenteritis and ileocolic intussusception (1). However, the causes of acute abdominal pain in children can also be of extra-abdominal origin, of which pneumonia is the most prominent one $(2,3)$. Pneumonia can also be found as an "accidental" finding in other conditions that demand an abdominal US evaluation. Accurate and prompt diagnosis of pneumonia is essential to implement early antibiotic therapy and prevent its complications, such as pleural effusion, necrosis and abscess formation (2). Although US was for a long time considered inadequate for the evaluation of pathological changes within the lungs, numerous studies over the last two decades have shown that US is not only an entirely valid method in the diagnostics of lung diseases, but in the diagnostics of pneumonia 
even more sensitive compared to chest X-ray (CXR) in the adult, and especially in the pediatric population (4-10).

While performing an abdominal US it is possible to transhepatically and transsplenically visualize both lung bases, as well as the pathological changes within them. However, during the US examination of the abdomen, the lung bases are often not in the primary focus of the investigator, so a positive finding can be overlooked, especially if the referral diagnosis does not suggest the existence of pulmonary pathology, and/or if the ultrasonographer is not experienced enough.

This study represents the experience of a single pediatric radiologist at a regional children's hospital. Its aim is to investigate the frequency of positive findings within the lung bases during abdominal US examinations in children with referral diagnosis not suggesting pulmonary disease, and to emphasize the importance of careful examination of both lung bases while performing abdominal US in children.

\section{Material and methods}

A retrospective study was conducted at the regional children's hospital, comprising a two-year period (1 Jan 2013 - 31 Dec 2014). The data for the number of abdominal US exams, both outpatient and inpatient, was retrieved from the Radiology Department protocols. In addition, the data for referral diagnoses under which the children were sent to abdominal US examinations were also retrieved from the former. Regarding the study group, an inclusion criterion was for the patients to have positive findings on lung bases visualized during the abdominal US, while the referral diagnosis did not suggest lung pathology.

A positive finding within the lung bases was detected using both the trans-hepatic

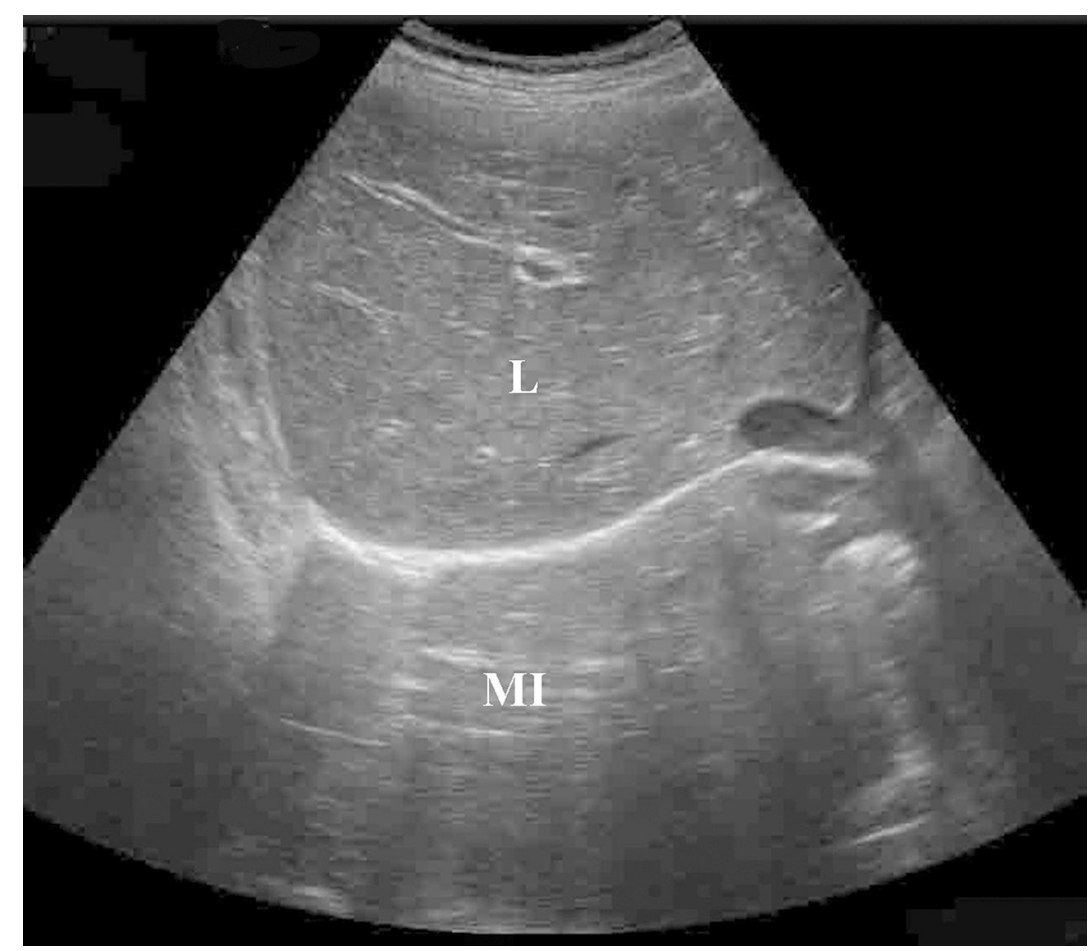

Fig. 1 Normal US finding of the right lung base transhepatically - supradiaphragmatic projection of the liver (,mirror image“ (MI) phenomenon). L - liver. 
and trans-splenic approach. All of the US examinations were conducted using Acuson S2000 and X300 (Siemens, Erlangen, Germany) ultrasound devices, with $5 \mathrm{MHz}$ convex, and $9 \mathrm{MHz}$ and $7.5 \mathrm{MHz}$ linear probes. In patients with positive trans-abdominal US findings within the lung bases, the transthoracic US approach to the lungs and pleura was also used.

The normal finding was visualized as a supradiaphragmatic projection of the liver or spleen (Fig. 1), which is based on the "mirror image" phenomenon $(11,12)$. The criteria for abnormal ultrasound findings within the pulmonary bases were: the existence of a consolidation of the lung parenchyma, pleural effusion, alveolar-interstitial edema (area of compact B lines), or combinations thereof. Vertically oriented "comet-tail" artifacts, called B lines, in trans-abdominal US appearance of lung bases, spread from the hemidiaphragm to the bottom of the screen, are hyperechogenic and clearly defined (Fig. 2).
They are a result of the accumulation of fluid in the subpleural interlobular septa surrounded by air $(13,14)$. A pneumonia-positive was considered each US finding of subpleural consolidation with air-bronchogram (Fig. 3), as well as consolidation with adjacent area of $B$ lines, or focal area of compact B lines. In children with positive US finding within the lung bases, in which the pediatrician or pediatric surgeon indicated CXR, we compared US and CXR findings, if the time interval between these diagnostic procedures was not longer than 2 hours. The most common referral diagnosis for outpatients and inpatients with abdominal US was found.

In the study group the prevalence of different referral diagnoses, as well as the distribution of the most common intra-abdominal pathological findings were calculated. In this group of patients, distribution of different lung base pathological findings was detected as well. The frequency of positive findings within the lung bases during abdominal US

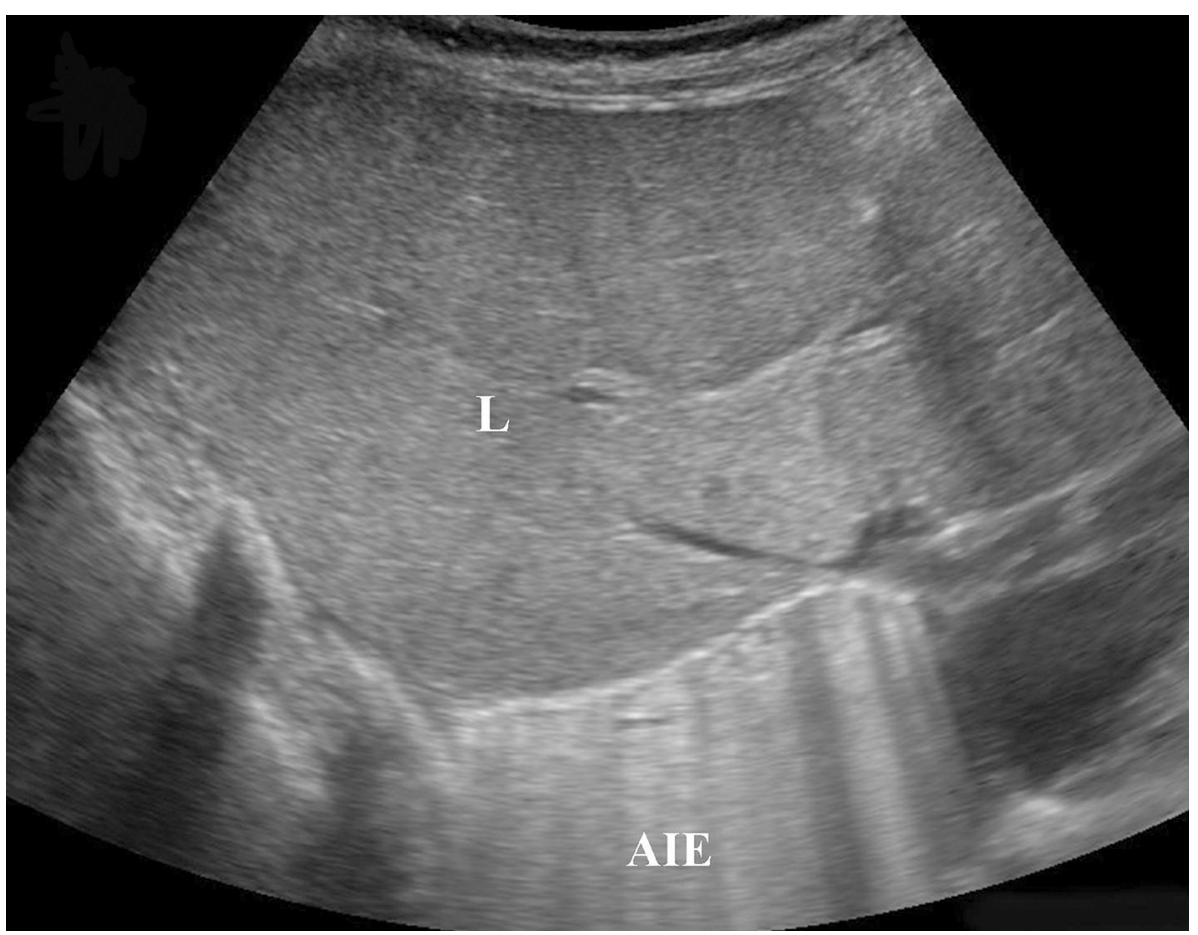

Fig. 2 Compact pattern of hyperechogenic B lines observed transhepatically within the right lung base, representing an alveolar-interstitial edema (AIE). L - liver. 


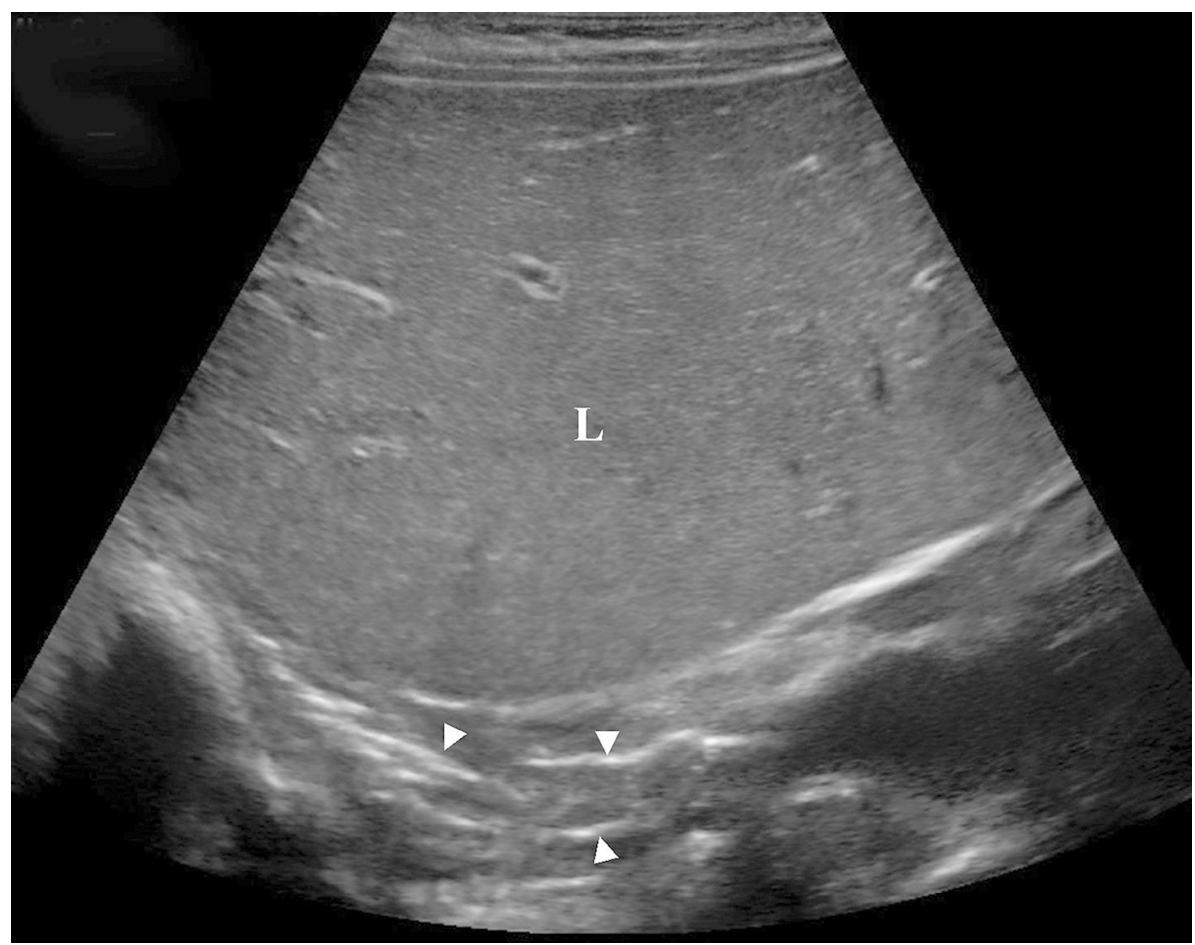

Fig. 3 Transhepatic approach to the right pulmonary base, within which a consolidation of lung parenchyma is seen, with hyperechogenic linear structures corresponding to the air bronchogram (arrow heads). L-liver.

in the group of outpatients, and in the group of outpatients with abdominal pain was calculated.

\section{Ethics statement}

The Ethical Committee of the Institute for Children and Adolescents Health Care of Vojvodina has approved the research (date of issue: 31 Jan 2015, registration number: 210/1-11). Its work conforms to the provisions of the Declaration of Helsinki in 1995 (as revised in Tokyo 2004).

\section{Results}

During the two-year period, one pediatric radiologist performed 1509 abdominal US examinations in the inpatient children and 2979 US in the outpatient children, which is 4488 abdominal US examinations in total in 2013 and 2014. Abdominal pain was the most common referral diagnosis - in 1469 examined patients (1257 outpatients, 212 inpatients), or $32.7 \%$ of all abdominal US examinations.

Over the two-year period, in 22 children aged 5 months to 17 years (average age 7.4 years, $\mathrm{SD}=4.2$ years, 15 males and 7 females) with abdominal US examination performed, positive US findings within the lung bases were found, for whom referral diagnoses did not suggest the existence of pathology originating from the respiratory tract. The referral diagnoses for this group of children were: abdominal pain (15 children), nausea and vomiting (3), febrile convulsions (2), exacerbation of juvenile arthritis (1), and in one child routine follow-up US of the abdomen and pelvis was performed the day after the 
successful hydrostatic reduction of ileocolic intussusception.

While performing the US examinations of the abdomen the following pulmonary pathologies were detected: 19 pneumonias (Fig.
4, 5) and 3 pneumonias complicated with pleural effusion. Of the positive abdominal US findings within the study group, the following stand out: splenomegaly (14 children), mesenteric lymphadenitis (13), ascites
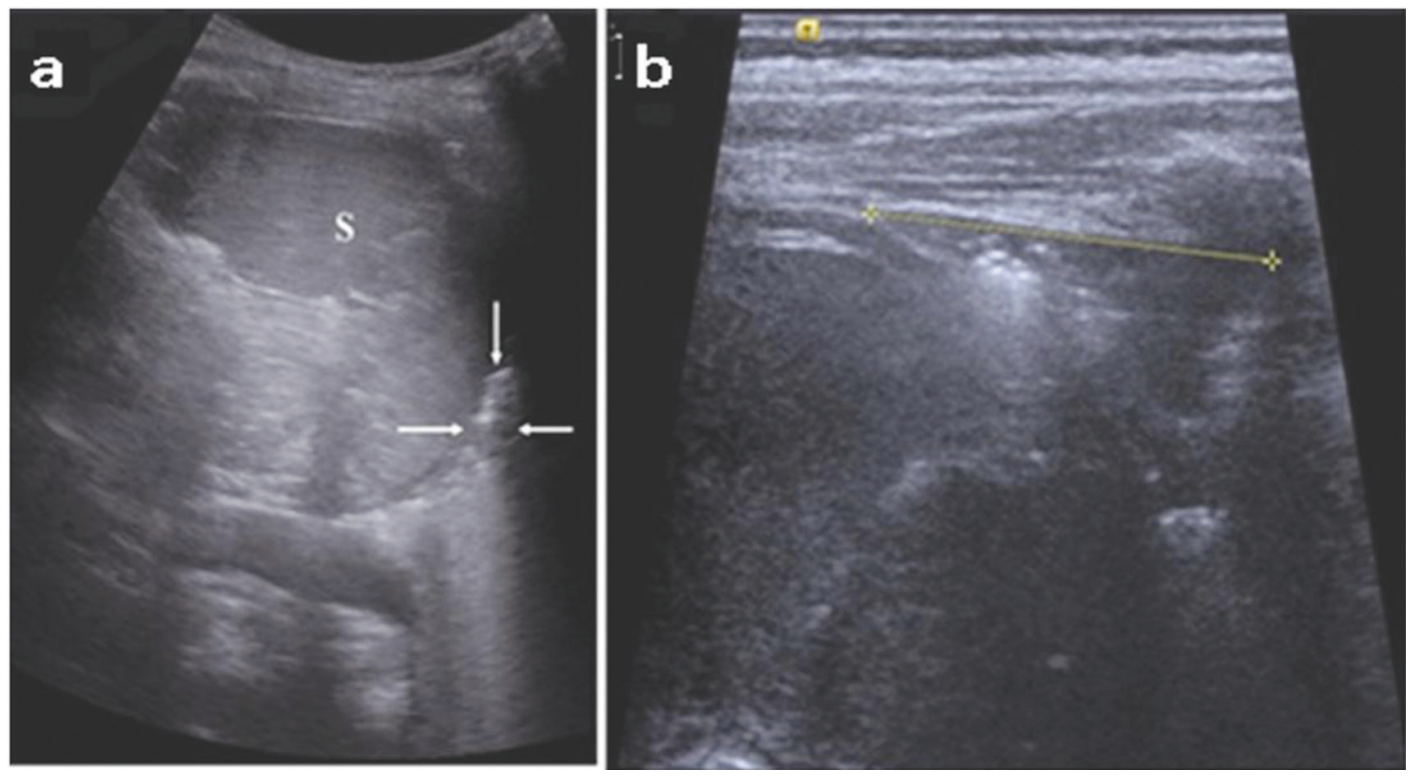

Fig. 4 A 2-year-old boy hospitalized because abdominal US revealed ileocolic intussusception, which was taken care by hydrostatic reduction under US control. A day after, follow-up abdominal US transsplenically (a) showed consolidation of the lung parenchyma within the left lung base (arrows), confirmed transthoracically (b) (marked with calipers, cranio-caudal diameter of $28 \mathrm{~mm}$ ). S-spleen.
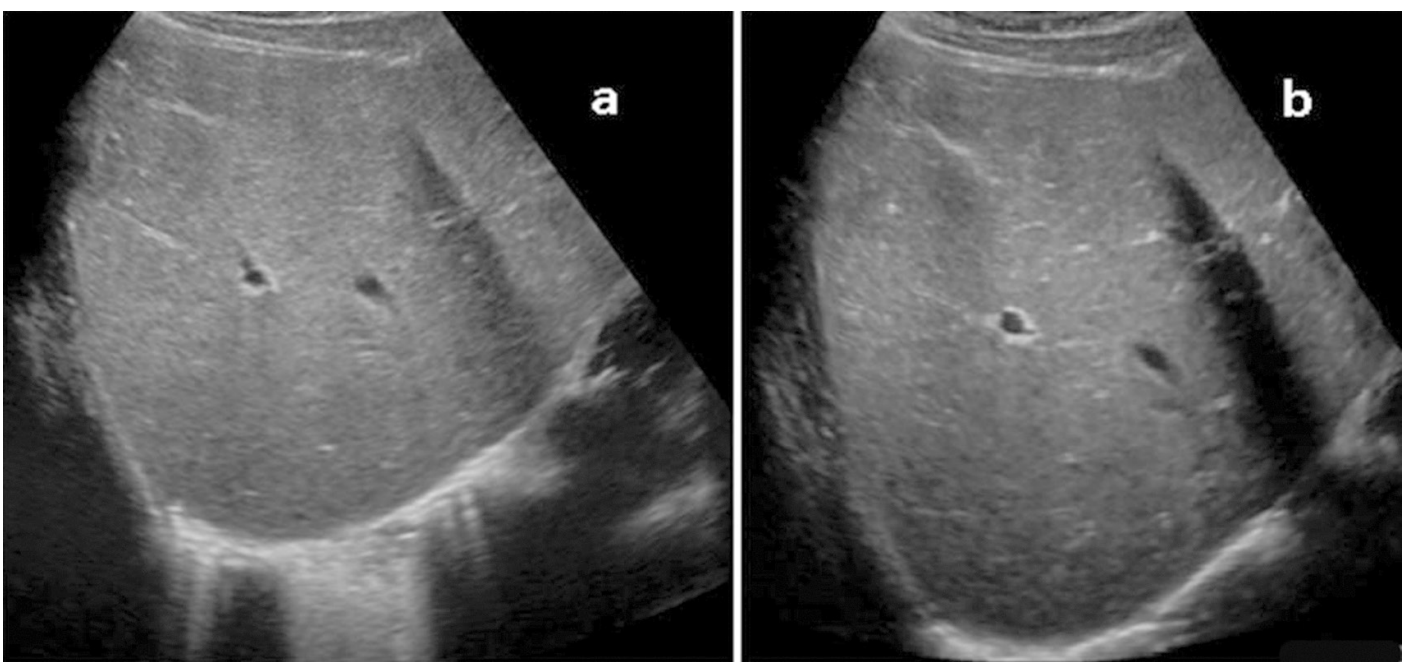

Fig. 5 (a) Transhepatically diagnosed pneumonic consolidation with LL diameter of $32 \mathrm{~mm}$, during abdominal US examination in a 7-year-old boy with abdominal pain; (b) Shows how changes within the lung bases may be overlooked with the adjustment of US parameters, in this case by reducing the depth of penetration of the ultrasound waves strictly to the field of interest, with the intention to more clearly visualize the liver. 


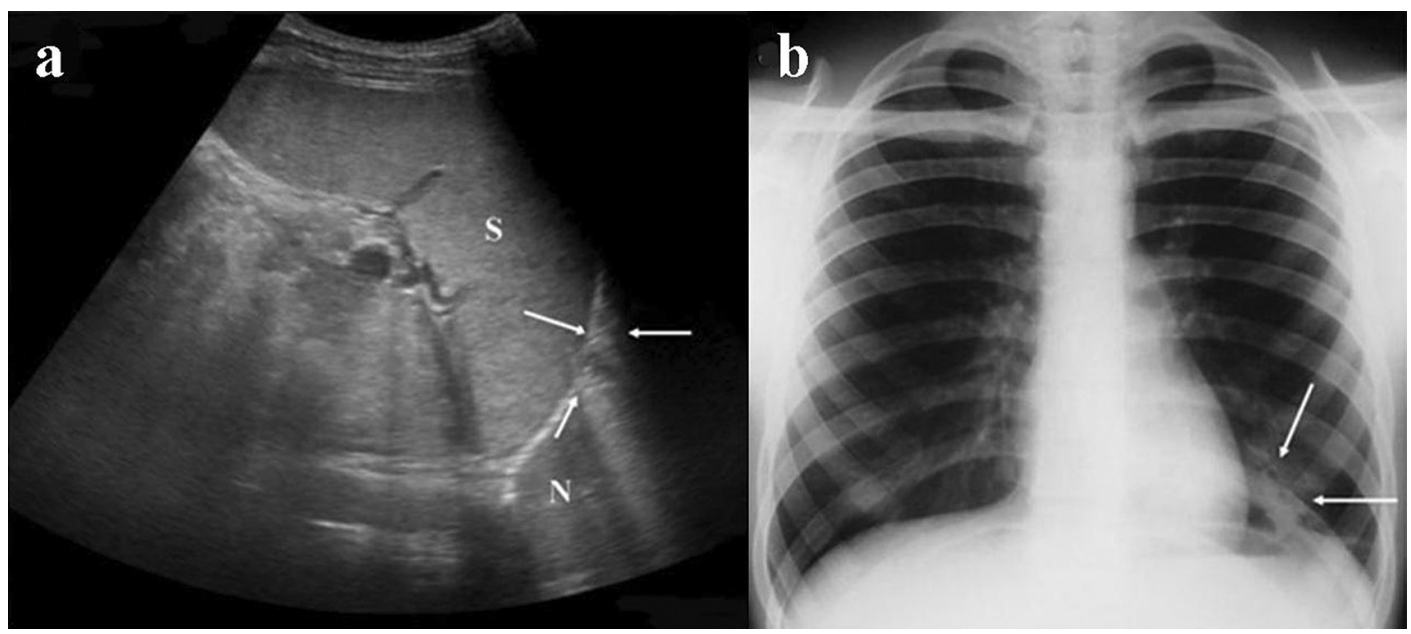

Fig. 6 A 13-year-old boy came as an outpatient to the Pediatric Emergency Department with abdominal pain and fever; (a) - During abdominal ultrasound a small area of consolidation (arrows) with the surrounding $B$ lines was detected transsplenically. $N$-area of normal US finding of the left lung base, $S$ - spleen. Transthoracically this consolidation has not been observed. (b) - Left-sided pneumonia was confirmed on CXR (arrows).

(6), and appendicitis (2), or the combination thereof. Of the 15 children with the referral diagnosis of abdominal pain, $10(66.7 \%)$ had mesenteric lymphadenitis.

Of the 22 children with positive lung base US findings, 18 were outpatients and 4 were inpatients. For every 165 outpatients one had a positive lung base US finding for pneumonia/pleuropneumonia, which amounts to $0.6 \%$. Of the 1257 examined outpatients with referral diagnosis of abdominal pain, 12 had positive lung base US findings for pneumonia/pleuropneumonia, i.e. 1 in every 105 examined patients, or $0.95 \%$. Three patients underwent CXR, and lung US findings were confirmed for all of them. In one patient the finding was positive only transabdominally, i.e. transsplenically, while the trans-thoracic approach showed a normal finding (Fig. 6).

\section{Discussion}

Lung and pleura ultrasonography is emerging as a complementary method in recent years, primarily to CXR, but, in some segments, even to CT, which is the golden standard in the diagnostics of pulmonary diseases
(4-10, 13, 15-20). However, this method is still not regularly applied in a large number of diagnostic centers.

In the course of the US examination of the abdomen, the trans-abdominal evaluation of costophrenic angles is already recognized, i.e. the eventual presence of pleural effusion in them, but often, in everyday clinical practice, the great potential of the evaluation of pathological changes within the lung parenchyma of the lung bases is ignored. The aim of this research was to point out the importance of the trans-hepatic and trans-splenic evaluation of lung bases when performing the abdominal US.

Trans-abdominal US examination of the lung bases is based on the acoustic phenomenon of "mirror image" $(11,12)$. The ultrasound waves which are transmitted through the tissues are subject to reverberation (reflection), refraction, dispersion and absorption. The number and proportion of the occurrences of these physical phenomena depends on the type of tissue, the frequency of ultrasound waves, the ratio between the wavelength and size of the target object, the object surface orientation in space and the acoustic 
resistance of the medium through which the ultrasound beam passes through (12). In children with normally aerated lungs, there is a full reflection of the ultrasound beam when reaching the boundary between the diaphragm and the lungs. After another reverberation on the liver parenchyma or spleen, US waves are transferred back to the phreno-pulmonary border and then returned to the US probe. The receivers in the probe cannot reconstruct the exact route of the US beam transmission, so the "lesion" is localized above the diaphragm, at a distance that corresponds to the echo return time. Therefore, a supradiaphragmatic projection of the liver or spleen is seen.

When the parenchymal disease propagates to the pleura, an acoustic window is formed and this creates a transmission of an ultrasound beam, enabling the evaluation of lung tissue (13). Pulmonary consolidations, hence, may be detected transabdominally only if in contact with basal pleura (12). During the two years of work of a single pediatric radiologist, it was noted that abnormal findings within the lung bases were found in 22 patients, in whom the referral diagnosis did not in any way suggest the existence of a pulmonary pathology. Most of the referral diagnoses in this group of children concerned abdominal pain, and pneumonia is, in several studies, referred to as the most common extra-abdominal cause of the abdominal pain $(2,3,21)$. Our study revealed $0.95 \%$ prevalence of pneumonia in outpatients with abdominal pain, which is lower than it is reported in the literature $-1.6 \%$ to $2.2 \%$ (2, 22-24). The reason for this might be the fact that by the US trans-abdominal approach it was possible to detect only pneumonic areas which abutted the basal pleura. Diagnosis of pneumonia in these children is of particular importance in everyday clinical practice, since it puts a physician-clinician on the right track for treatment, i.e. it contribudes to the timely diagnosis and treatment of these children, as well as prevents the development of complications (pleural effusion, necrosis, abscess) which prolong the treatment period, increase the number of hospital days, treatment costs, are treated by combinations of several antibiotics, and, most importantly, in some cases, they can have lasting effects. Naturally, with timely diagnosis the number of negative laparotomies should decrease (2). The greatest number of children in our study had splenomegaly along with pneumonia, as well as mesenteric lymphadenitis, which is considered one of the most common causes of abdominal pain in children $(21,25)$. Additionally, one of the causes of abdominal pain is irritation of the diaphragmatic, basal pleura, which was probably the cause of pain in three of the children with pleuropneumonia.

Pneumonia can, on occasion, be found accompanying serious, urgent abdominal pathology, which in our research was the case with three children ( 2 appendicitises and 1 ileocolic intussusception). The existence of pneumonia (2 patients) or pleuropneumonia (1 patient) in these children had a significant impact on the type and duration of the antibiotic therapy applied. Generally, in all patients with trans-abdominal US diagnosed pneumonia/pleuropneumonia in the region of lung bases, this finding brought a new and extremely important clinical information that had significantly influenced the clinical course and faster recovery of these patients. A few studies showed that lung ultrasound often provides clinicians with new information of great value for the treatment of patients, compared to other diagnostic methods (8, 13, 26).

Our hypothesis that radiologists do not always pay enough attention to the pulmonary bases was the most clearly demonstrated in a child with intussusception. The initial US examination showed the existence of ileocolic intussusception, which was taken care 
the immediately after the diagnosis (hydrostatic reduction under US control), but not the existence of left-sided pneumonia, which was diagnosed at the follow-up US after less than 15 hours. Of course, hypothetically, it is possible that it developed during these 15 hours, but it still seems more probable that pneumonia existed. However, in searching for emergency abdominal surgery diagnosis, simply not enough attention was devoted to the evaluation of lung bases. The initial and follow-up US examinations were performed by different pediatric radiologists. In the great desire of radiologists to get a better, "full screen" display of liver and spleen for their better and more accurate evaluation, it is easy to overlook the important pathological changes within the lung bases.

In one child, our study showed that a finding within the lungs can be positive only transabdominally and completely negative transthoracically. Considering that the performer of the US examinations is an experienced pediatric radiologist in the field of lung US diagnostics, lack of experience cannot be blamed for the absence of trans-thoracic pneumonia visualization. This indicates that low trans-thoracic approach and the trans-abdominal approach are not the same, although often identified as such. Two of our previous studies have shown that transabdominal findings can be more extensive compared to the trans-thoracic, but so far we have not had the situation where positive US findings were detected using trans-splenic approach, whereas trans-thoracic approach showed a normal LUS pattern $(8,27)$. We have noticed this situation only once on the right side, using trans-hepatic approach, and described the reason for it (28). At this point, we do not have an explanation for this phenomenon within the left lung base, but we will search for it in the future studies.

This study has several limitations. In the available literature databases, studies dealing with this kind of research were not found. The possibility of comparing data from this survey with data from other studies was limited, because only those with somewhat similar topics were available. It was not possible to re-review previous abdominal US examinations in children with short interval follow-ups because we do not have a system for saving all the US images of every child, but only print a few specific images. Therefore, we cannot determine the reason why the lesions were not identified on the initial US exam (incomplete inclusion of the lung bases, interpretation error?). Also, CXRs were not performed in all children with the US detected pneumonia, which depended on the clinical practitioner's decision. Lung ultrasound is widely excepted in our hospital, and most of the time, when the US finding is pneumonia-positive, CXR is avoided, especially when the US finding is supported by clinical finding and laboratory data.

\section{Conclusion}

In conclusion, every radiologist should be aware of the potential pathological findings within the lung bases, which can be detected by carefully performed ultrasound examination of the abdomen. These findings are not very common, but can have a very significant impact on the clinical course and treatment of the child. The trans-abdominal ultrasound approach might sometimes provide additional information about the lung bases, even when compared to a standard trans-thoracic approach. The protocol for the abdominal ultrasound examination, especially in children with acute abdominal pain, should include the examination of the lung bases.

\section{What is already known on this subject}

Lung ultrasound is already recognized as an extremely useful diagnostic tool in detecting pneumonia in every day pediatric clinical practice. The causes of acute abdominal pain in chil- 
dren can be of extra-abdominal origin, of which pneumonia is the most prominent one.

\section{What this study adds}

This study brings attention to the need of careful examination of lung bases while performing abdominal ultrasound, especially for patients with abdominal pain. It can have a serious impact on the treatment of children. In the available literature we could not find the study dealing with this particular topic.

Authors' contributions: Conception and design: JL, DP; Acquisition, analysis and interpretation of data: JL, DP, IV, SP; Drafting the article: JL; Revising the article critically for intellectual content: JL, SP; Approved final version of the manuscript: JL, DP, IV, SP.

Conflict of interest: The authors declare that they have no conflict of interest.

\section{References}

1. Kim JS. Acute abdominal pain in children. Pediatric gastroenterology, hepatology \& nutrition. 2013;16(4):219-24.

2. Ravichandran D, Burge DM. Pneumonia presenting with acute abdominal pain in children. $\mathrm{Br} \mathrm{J}$ Surg. 1996;83(12):1707-8.

3. Tsalkidis A, Gardikis S, Cassimos D, Kambouri K, Tsalkidou E, Deftereos S, et al. Acute abdomen in children due to extra-abdominal causes. Pediatr Int. 2008;50(3):315-8.

4. Blaivas M. Lung Ultrasound in Evaluation of Pneumonia. J Ultrasound Med. 2012;31(6):823-6.

5. Caiulo VA, Gargani L, Caiulo S, Fisicaro A, Moramarco F, Latini G, et al. Lung ultrasound characteristics of community-acquired pneumonia in hospitalized children. Pediatr Pulmonol. 2013;48(3):280-7.

6. Copetti R, Cattarossi L. Ultrasound diagnosis of pneumonia in children. Radiol Med. 2008;113(2):190-8.

7. Cortellaro F, Colombo S, Coen D, Duca PG. Lung ultrasound is an accurate diagnostic tool for the diagnosis of pneumonia in the emergency department. Emerg Med J. 2012;29(1):19-23.

8. Lovrenski J, Petrović S, Varga I, Varga J. Pneumonias in children - comparison of lung ultrasonography findings with chest X-rays. Paediatr Croat. 2013;57(3):227-34.

9. Parlamento S, Copetti R, Di Bartolomeo S. Evaluation of lung ultrasound for the diagno- sis of pneumonia in the ED. Am J Emerg Med. 2009;27(4):379-84

10. Reissig A, Copetti R, Mathis G, Mempel C, Schuler A, Zechner P, et al. Lung ultrasound in the diagnosis and follow-up of community-acquired pneumonia: a prospective, multicenter, diagnostic accuracy study. Chest. 2012;142(4):965-72.

11. Avni EF, Braude P, Pardou A, Matos C. Hyaline membrane disease in the newborn: diagnosis by ultrasound. Pediatr Radiol. 1990;20(3):143-6.

12. Cosgrove DO, Garbutt P, Hill CR. Echoes across the diaphragm. Ultrasound Med Biol. 1978;3(4):385-92.

13. Lichtenstein DA, Mezière GA. Relevance of lung ultrasound in the diagnosis of acute respiratory failure: the BLUE protocol. Chest. 2008;134(1):11725.

14. Lichtenstein D, Mezière G. A lung ultrasound sign allowing bedside distinction between pulmonary edema and COPD: the comet-tail artifact. Intensive Care Med. 1998;24(12):1331-4.

15. Bourcier J-E, Paquet J, Seinger M, Gallard E, Redonnet JP, Cheddadi F, et al. Performance comparison of lung ultrasound and chest $\mathrm{x}$-ray for the diagnosis of pneumonia in the ED. Am J Emerg Med. 2014;32(2):115-8.

16. Khalil M, Diab HS, Hosny H, Edward E, Thabet E, Emara W, et al. Chest ultrasound versus chest computed tomography for imaging assessment before medical thoracoscopy. Egypt J Bronchol. 2014;8:149-52.

17. El Sheikh H, Abd Rabboh MM. Chest ultrasound in the evaluation of complicated pneumonia in the ICU patients: Can be viable alternative to CT? The Egyptian Journal of Radiology and Nuclear Medicine. 2014;45(2):325-31.

18. Kurian J, Levin TL, Han BK, Taragin BH, Weinstein S. Comparison of ultrasound and CT in the evaluation of pneumonia complicated by parapneumonic effusion in children. AJR Am J Roentgenol. 2009;193(6):1648-54.

19. Nazerian P, Volpicelli G, Vanni S, Gigli C, Betti L, Bartolucci M, et al. Accuracy of lung ultrasound for the diagnosis of consolidations when compared to chest computed tomography. Am J Emerg Med. 2015;33(5):620-5.

20. Shah VP, Tunik MG, Tsung JW. Prospective evaluation of point-of-care ultrasonography for the diagnosis of pneumonia in children and young adults. JAMA Pediatr. 2013;167(2):119-25. 
21. Moustaki M, Zeis PM, Katsikari M, Fretzayas A, Grafakou O, Stabouli S, et al. Mesenteric lymphadenopathy as a cause of abdominal pain in children with lobar or segmental pneumonia. Pediatr Pulmonol. 2003;35(4):269-73.

22. Homier V, Bellavance C, Xhignesse M. Prevalence of pneumonia in children under 12 years of age who undergo abdominal radiography in the emergency department. CJEM. 2007;9(5):347-51.

23. Jones PF. Active observation in management of acute abdominal pain in childhood. Br Med J. 1976;2(6035):551-3.

24. Sims DG, Alexander FW. Acute abdominal pain in childhood. Br Med J. 1976;2(6040):880-1.

25. Vayner N, Coret A, Polliack G, Weiss B, Hertz M. Mesenteric lymphadenopathy in children exam- ined by US for chronic and/or recurrent abdominal pain. Pediatr Radiol. 2003;33(12):864-7.

26. Yuan A, Yang PC, Chang YC, Kuo SH, Luh KT, Chen WJ, et al. Value of chest sonography in the diagnosis and management of acute chest disease. J Clin Ultrasound. 2001;29(2):78-86.

27. Lovrenski J. Lung ultrasonography of pulmonary complications in preterm infants with respiratory distress syndrome. Ups J Med Sci. 2012;117(1):107.

28. Lovrenski J, Petrović S, Balj-Barbir S, Jokić R, Vilotijević-Dautović G. Stethoscope vs. ultrasound probe - which is more reliable in children with suspected pneumonia? Acta Med Acad. 2016;45(1):39-50. 